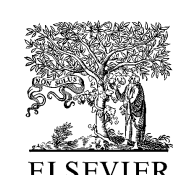

\title{
Incremental prognostic value of troponin I and echocardiography in patients with acute pulmonary embolism
}

\author{
Nils Kucher*, Dieter Wallmann, Angelo Carone, Stephan Windecker, \\ Bernhard Meier, Otto Martin Hess
}

Cardiology, Swiss Cardiovascular Center, University Hospital, 3010 Bern, Switzerland

Received 24 January 2003; received in revised form 24 June 2003; accepted 26 June 2003

\section{KEYWORDS \\ Pulmonary embolism; prognosis; echocardiography}

\begin{abstract}
Background To test the hypothesis that troponin I and echocardiography have an incremental prognostic value in patients with pulmonary embolism (PE).

Methods and results In 91 patients with acute PE, echocardiography was performed within $4 \mathrm{~h}$ of admission. Troponin I levels were obtained on admission and $12 \mathrm{~h}$ thereafter. The $0.06 \mu \mathrm{g} / \mathrm{l}$ troponin I cut-off level was identified as the most useful, high-sensitivity cut-off level for the prediction of adverse outcome by receiver operating characteristic analysis with a sensitivity and specificity of $86 \%$, respectively. Twenty-eight (31\%) patients had elevated troponin I levels $(4.9 \pm 3.8 \mu \mathrm{g} / \mathrm{l})$. Twenty-one (23\%) patients had adverse clinical outcomes including in-hospital death in five, cardiopulmonary resuscitation in four, mechanical ventilation in six, pressors in 14, thrombolysis in 14, catheter fragmentation in three, and surgical embolectomy in three. The area under the receiver operating characteristic curve from multivariate regression models for predicting adverse outcome without troponin I and echocardiography $(0.765)$, with troponin I $(0.890)$ or echocardiography alone $(0.858)$, and the combination of both tests $(0.900)$ was incremental. Three-month survival rate was highest in patients with both a normal troponin I level and a normal echocardiogram (98\%). Positive predictive value for adverse clinical outcomes of the combination of echocardiography and troponin I was higher $(75 \%(95 \% \mathrm{Cl} 55-88 \%))$ compared with each test alone (echocardiography: $41 \%$, $95 \% \mathrm{Cl} 28-56 \%$; troponin I: 64\%, 95\% Cl 46-79\%).

Conclusions While troponin I measurements added most of the prognostic information for identifying high-risk patients, a normal echocardiogram combined with a negative troponin I level was most useful to identify patients at lowest risk for early death. (c) 2003 Published by Elsevier Ltd on behalf of The European Society of Cardiology.
\end{abstract}

\section{Introduction}

Right ventricular dysfunction was the single most important predictor of in-hospital death in the international registry of 2454 patients with pulmonary embolism (PE). ${ }^{1}$ Therefore, echocardiography has emerged as important

\footnotetext{
* Correspondence to: Nils Kucher, MD, Cardiovascular Division, VTE Research Group, Brigham and Women's Hospital, Harvard Medical School, 75 Francis Street, Boston, MA 02115, USA. Tel: +1 61773269 86; fax: +1 6177387652

E-mail address: nkucher@partners.org (N. Kucher).
}

diagnostic tool for assessing the degree of right ventricular dysfunction in patients with acute PE.

Recently, cardiac troponins I and $\mathrm{T}$ have also been shown to be associated with early mortality and a complicated hospital course in patients with PE. ${ }^{2-4}$ However, the prognostic role of cardiac troponins in PE patients who undergo risk stratification, including assessment of right ventricular function by echocardiography, is less clear. We aimed to test the hypothesis that troponin I and echocardiography have an incremental prognostic value in $\mathrm{PE}$ patients. 


\section{Methods}

\section{Patients}

In consecutive symptomatic PE patients from the Emergency Department of the University Hospital of Bern, the diagnosis was confirmed by spiral computed tomography (CT) in 86, high probability ventilation perfusion scan in three, and pulmonary angiography in two patients during a study period of 18 months. Troponin I levels were taken in all 91 patients on admission and $12 \mathrm{~h}$ thereafter using a microparticle enzyme immunoassay (Abbott, USA). The study protocol was approved by the local ethics committee, and written informed consent was obtained from all patients.

\section{Spiral computed tomography, ventilation perfusion scan, and pulmonary angiography}

Pulmonary embolism was diagnosed by spiral CT when there was at least one intravascular filling defect in a pulmonary artery using a standard protocol. ${ }^{5}$ Lung scans were interpreted according to the PIOPED criteria. ${ }^{6}$ PE was diagnosed by pulmonary angiography when there was at least one intravascular filling defect in a pulmonary artery using a standard protocol. ${ }^{7}$

\section{Transthoracic echocardiography}

Echocardiography was performed in all patients with PE within $4 \mathrm{~h}$ after admission using an Acuson Sequoia ${ }^{\mathrm{TM}} \mathrm{C} 256$ system (Mountain View, California, USA) with a $3.5 \mathrm{MHz}$ probe and three-lead electrocardiographic monitoring. Echocardiographic off-line analysis was interpreted by a cardiologist who was unaware of clinical data and troponin I levels. Right ventricular end-diastolic diameter was measured either from the apical or subcostal four-chamber view in $85(93 \%)$ patients. The maximal distance between the endocardium of the right ventricular free wall and the interventricular septum, perpendicular to the long axis of the ventricle, was measured at the beginning of the QRS-complex. Tricuspid pressure gradient as a surrogate of systolic pulmonary artery pressure was available in 70 (77\%) patients. Right ventricular systolic function was assessed in all patients using a 4-point scale, i.e., normal/near normal right ventricular systolic function, moderate to severe right ventricular systolic dysfunction. ${ }^{8,9}$

\section{Treatment strategy}

Reperfusion therapy, including thrombolysis, catheter fragmentation, or surgical embolectomy was performed in patients with $\mathrm{PE}$ and a shock index $\geq 1$ (heart rate divided by systolic blood pressure). ${ }^{10}$ Thrombolysis was also considered in the absence of contraindications according to the Task Force of the European Society of Cardiology (ESC) ${ }^{11}$ in patients with a shock index $<1$ but moderate to severe right ventricular dysfunction. All patients with normal/near normal right ventricular function on the echocardiogram were treated with heparin alone.

\section{Statistical analysis}

Adverse clinical outcome was defined as in-hospital death or the need for cardiopulmonary resuscitation, mechanical ventilation, pressors, thrombolysis, catheter fragmentation, or surgical embolectomy according to the MAPPET- 3 criteria. ${ }^{12}$

Table 1 and Table 2 data are presented as numbers of patients with proportions in parentheses or mean \pm SD values.
Receiver operating charcteristic (ROC) analysis was performed to identify the most useful troponin I cut-off level for the prediction of adverse outcome. Nominal data comparison between the troponin groups (cut-off $0.06 \mu \mathrm{g} / \mathrm{l}$ ) was performed using a $\chi^{2}$ test. Continuous echocardiographic and haemodynamic data between the troponin groups were compared with the Student t-test.

Multivariate logistic regression was performed on univariately significant continuous and categorical variables $(P<0.05)$ from Table 1 for predicting adverse clinical outcomes. To investigate whether troponin I and echocardiography have incremental prognostic value in addition to clinical parameters, we also calculated resulting areas under the ROC curve for multivariate models without troponin and echocardiography, with troponin I and echocardiography alone, and with the combination of both tests. All models were adjusted for univariately significant $(P<0.05)$ predictors of adverse outcome from Table 1.

Kaplan-Meier analyses were performed to investigate cumulative 3-month survival rates according to troponin I levels and the presence or absence of right ventricular dysfunction. Data were considered significant at $P<0.05$.

\section{Results}

None of the 91 patients had evidence for acute coronary syndromes, including right ventricular infarction, based on electrocardiograms, echocardiograms, and creatine kinase-MB levels. Twenty eight (31\%) patients had elevated troponin I levels $(4.9 \pm 3.8$, range $0.7-14.6 \mu \mathrm{g} / \mathrm{l})$. Syncope and dyspnea were more prevalent in patients with a troponin I leak than in patients with a normal troponin I level (Table 1). The history and risk factors for coronary artery disease were similar in patients with and without troponin I elevation. Haemodynamic changes were more severe and moderate to severe right ventricular dysfunction more frequent in patients with than without elevated troponin I levels. Right ventricular dilatation was pronounced, and tricuspid pressure gradients were also higher in patients with elevated troponin I levels. More patients had paracentral or central pulmonary artery filling defects as assessed by spiral CT in the elevated troponin I group.

In-hospital mortality was 5\%, and all five deaths were attributed to right ventricular failure. Four of these patients had elevated troponin I levels. Twenty one (23\%) patients had adverse clinical outcomes including cardiopulmonary resuscitation in four, mechanical ventilation in six, pressors in 14, thrombolysis in 14, catheter fragmentation in three, and surgical embolectomy in three patients. Detailed combinations of adverse clinical outcomes are shown in Table 2 The $0.06 \mu \mathrm{g} / \mathrm{l}$ troponin I cut-off level was identified as the most useful, highsensitivity cut-off level for the prediction of adverse outcome by receiver operating characteristic analysis with a sensitivity and specificity of $86 \%$, respectively.

Normal troponin I levels were associated with a higher 3-month survival rate than in patients with troponin I levels $\geq 0.06 \mu \mathrm{g} /$ ( Fig. 1). Three-month survival rate was highest in patients with both a normal troponin I level and a normal echocardiogram (Fig. 2).

Specificity and positive predictive value for adverse clinical events were higher using the combination of 
Table 1 Baseline characteristics of 91 patients with pulmonary embolism

\begin{tabular}{|c|c|c|c|}
\hline & Total & Troponin I $\geq 0.6 \mu \mathrm{g} / \mathrm{l}$ & Troponin I $<0.6 \mu \mathrm{g} / \mathrm{l}$ \\
\hline $\mathrm{n}$ & 91 & 28 & 63 \\
\hline Age, years & $61 \pm 17$ & $62 \pm 17$ & $61 \pm 18$ \\
\hline Men, $\mathrm{n}(\%)$ & 41 & $15(54)$ & $36(57)$ \\
\hline \multicolumn{4}{|l|}{ Symptoms, n (\%) } \\
\hline Dyspnoea & 40 & $25(89)^{\dagger}$ & $35(56)$ \\
\hline Chest pain & 38 & $13(46)$ & $25(40)$ \\
\hline Syncope & 12 & $9(32)^{\dagger}$ & $3(5)$ \\
\hline \multicolumn{4}{|l|}{ Comorbidities, n (\%) } \\
\hline Chronic lung disease & 11 & $3(11)$ & $8(13)$ \\
\hline Valvular heart disease & 4 & $1(4)$ & $3(5)$ \\
\hline Percutaneous coronary intervention & 4 & $1(4)$ & $3(5)$ \\
\hline Coronary artery bypass graft & 2 & $1(4)$ & $1(2)$ \\
\hline Previous myocardial infarction & 8 & $3(11)$ & $5(8)$ \\
\hline Angina pectoris & 6 & $2(7)$ & $4(6)$ \\
\hline Smoking & 19 & $7(25)$ & 12 (19) \\
\hline Hypercholesterolaemia & 22 & $8(29)$ & $14(22)$ \\
\hline Systemic hypertension & 21 & $7(25)$ & $14(22)$ \\
\hline Diabetes mellitus & 2 & 0 & $2(3)$ \\
\hline Family history for coronary artery disease & 8 & $3(11)$ & $5(8)$ \\
\hline \multicolumn{4}{|l|}{ Haemodynamic and echocardiographic data } \\
\hline Systolic blood pressure, $\mathrm{mmHg}$ & $120 \pm 17$ & $102 \pm 15^{*}$ & $127 \pm 17$ \\
\hline Heart rate, beats per minute & $85 \pm 15$ & $97 \pm 15^{*}$ & $81 \pm 16$ \\
\hline Shock index, bpm/mmHg & $0.71 \pm 0.17$ & $0.98 \pm 0.27^{*}$ & $0.65 \pm 0.18$ \\
\hline Right ventricular end-diastolic diameter, mm & $41 \pm 6$ & $48 \pm 6^{*}$ & $40 \pm 6$ \\
\hline Tricuspid systolic gradient, mmHg & $45 \pm 18$ & $57 \pm 16^{\dagger}$ & $43 \pm 18$ \\
\hline Moderate/severe right ventricular dysfunction, $\mathrm{n}(\%)$ & 46 & $24(86)^{*}$ & $22(35)$ \\
\hline Paracentral PE & 39 & $22(79)^{*}$ & $17(27)$ \\
\hline
\end{tabular}

Shock index: heart rate divided by systolic arterial blood pressure; Paracentral PE: $\geq 1$ subtotal filling defect in $\geq 1$ lobar or main pulmonary artery by spiral CT

${ }^{*} P<0.001$.

${ }^{\dagger} P<0.01$.

Table 2 Combinations of adverse clinical outcomes in 21 patients according to the presence or absence of a troponin I leak

\begin{tabular}{lll}
\hline Adverse clinical outcomes & Troponin $\geq 0.06 \mu \mathrm{g} / \mathrm{l}$ & Troponin $<0.06 \mu \mathrm{g} / \mathrm{l}$ \\
\hline Thrombolysis* & 2 & 1 \\
Pressors & 1 & 1 \\
Pressors+ventilation+death & 2 & - \\
Pressors+thrombolysis & 3 & - \\
Pressors+thrombolysis+death & 1 & 1 \\
Pressors+catheter fragmentation & 1 & - \\
Pressors+catheter fragmentation+thrombolysis & 2 & - \\
Surgical embolectomy & 1 & - \\
Pressors+ventilation+embolectomy & 1 & - \\
CPR+pressors+ventilation+embolectomy+death & 1 & - \\
CPR+thrombolysis & 1 & - \\
CPR+ventilation+thrombolysis & 1 & - \\
CPR+pressors+ventilation+thrombolysis+death & 1 & - \\
\hline
\end{tabular}

*Patients with right ventricular dysfunction and a shock index <1 (heart rate divided by systolic arterial blood pressure). CPR: cardiopulmonary resuscitation.

troponin I and echocardiography compared with that of each test individually (Table 3). Sensitivity and negative predictive value for adverse outcomes were similar for troponin I, echocardiography, and the combination of both tests.
In a multivariate regression model, troponin I elevation was an independent predictor of adverse outcome when adjusted for right ventricular function on the echocardiogram and other univariate predictors of adverse outcome (Table 4). The area under the ROC curve 


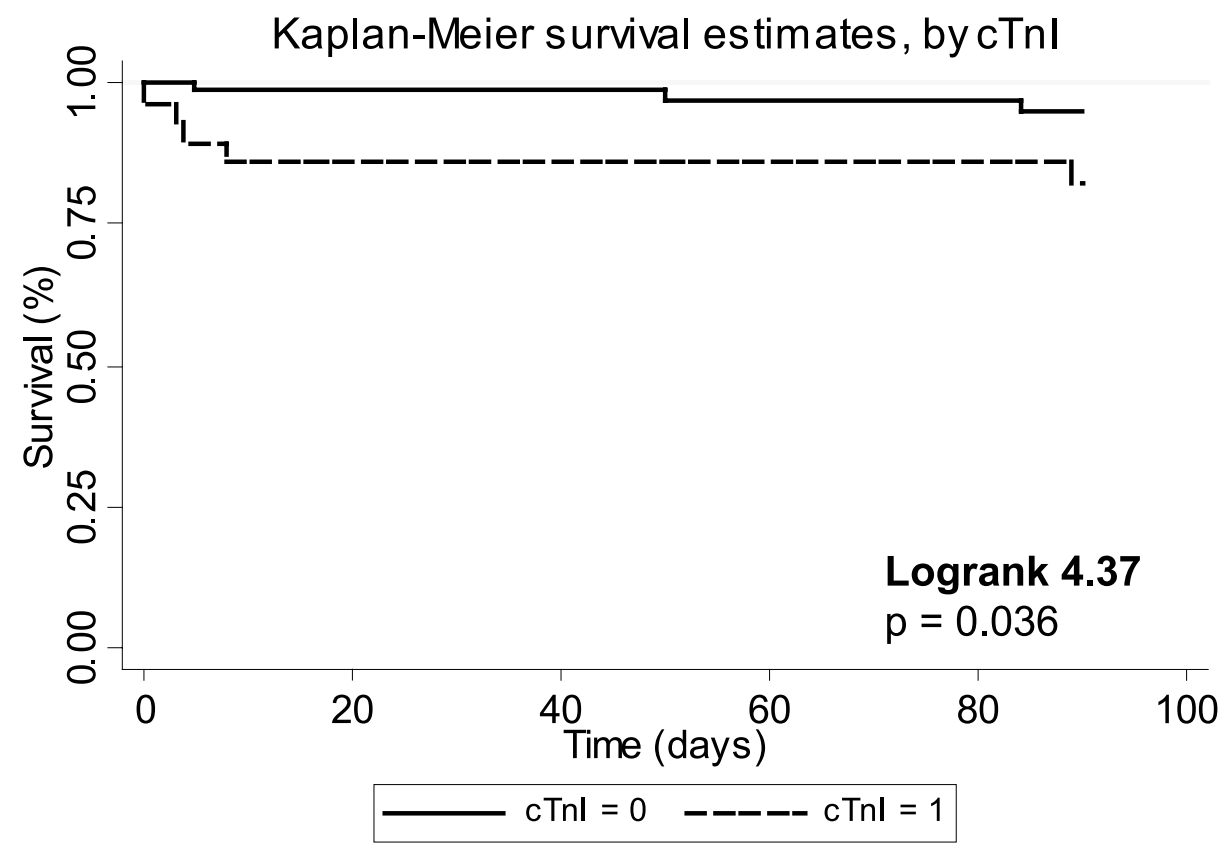

Fig. 1 Cumulative 90 -day survival rate in 91 patients with pulmonary embolism according to troponin I levels (cTnl cut-off $0.06 \mu$ g/l).

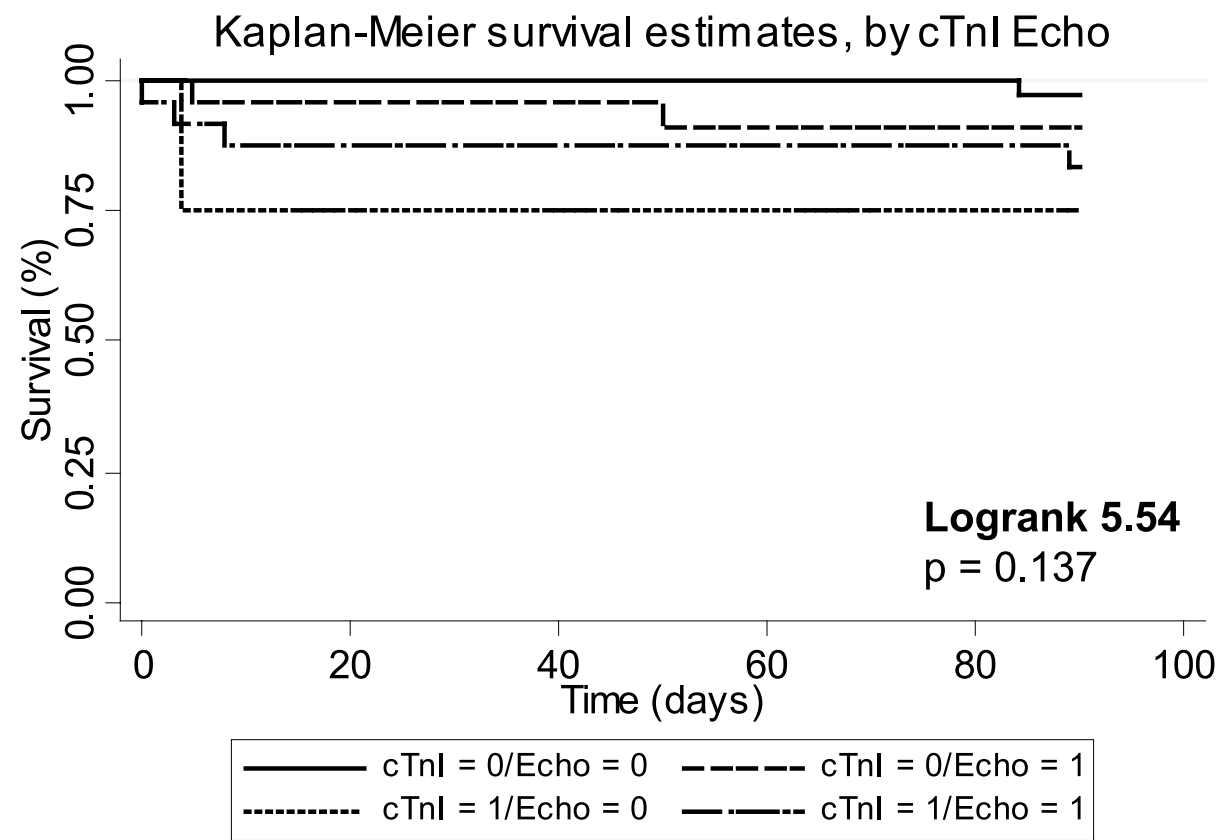

Fig. 2 Cumulative 90-day survival rate in 91 patients with pulmonary embolism based on troponin I levels (cTnl cut-off $0.06 \mu$ g/l) and right ventricular function on the echocardiogram.

obtained from multivariate regression models were: 0.765 for the model without troponin I and echocardiography, 0.890 for troponin I alone, 0.858 for echocardiography alone, and 0.900 for the combination of both tests.

\section{Discussion}

In the present study of 91 patients with acute PE, the combination of troponin I and echocardiography had ad- ditional prognostic information compared with troponin I or echocardiography alone. The area under the ROC curve from multivariate regression models for predicting adverse outcome without troponin I and echocardiography $(0.765)$, with troponin I $(0.890)$ or echocardiography alone $(0.858)$, and the combination of both tests $(0.900)$ was incremental. The biggest addition of prognostic information was obtained with troponin I measurements. Although the area under the curve did no increase much 
Table 3 Accuracy of troponin I and echocardiography for the prediction of adverse clinical outcomes

\begin{tabular}{llll}
\hline & Troponin I & Echocardiography & Troponin I+echocardiography \\
\hline Test positive, $\mathrm{n}(\%)$ & $28(31)$ & $46(50)$ & $24(26)^{*}$ \\
Specificity,\% $(95 \% \mathrm{Cl})$ & $86(76-92)$ & $61(50-72)$ & $91(83-96)$ \\
Positive predictive value,\% $(95 \% \mathrm{Cl})$ & $64(46-79)$ & $41(28-56)$ & $75(55-88)$ \\
Sensitivity,\% $(95 \% \mathrm{Cl})$ & $86(65-95)$ & $90(71-97)$ & $86(77-99)$ \\
Negative predictive value,\% $(95 \% \mathrm{Cl})$ & $95(87-98)$ & $96(85-99)$ & $96(88-98)$ \\
\hline
\end{tabular}

*Test positive: echocardiography positive (moderate to severe right ventricular dysfunction) and troponin I positive ( $\geq 0.6 \mu \mathrm{g} / \mathrm{l})$. Test negative: echocardiography and troponin I negative $(n=41)$, echocardiography positive and troponin I negative $(n=22)$, or echocardiography negative and troponin I positive $(n=4)$.

Table 4 Final multiple logistic regression model for the prediction of adverse clinical outcomes

\begin{tabular}{lll}
\hline Variable & $\begin{array}{l}\text { Adjusted OR } \\
(95 \% \mathrm{Cl})\end{array}$ & $P$ \\
\hline Troponin I & $1.7(1.2-2.4)$ & 0.003 \\
Moderate to severe right & $4.4(0.8-24.5)$ & 0.095 \\
ventricular dysfunction & $2.1(0.3-13.8)$ & 0.444 \\
Shock index & $1.4(0.3-7.8)$ & 0.667 \\
\hline Paracentral PE* &
\end{tabular}

* $\geq 1$ subtotal filling defect in $\geq 1$ lobar or main pulmonary artery by spiral CT.

with addition of echocardiography to troponin I results, it appears to be useful in identifying low-risk patients because patients with a normal troponin I level and a normal echocardiogram had the lowest mortality rate (Fig. 2). The positive predictive value and specificity for predicting adverse outcomes were higher with the combination of troponin I and echocardiography compared with each test alone (Table 3).

The prevalence of increased cardiac troponin I levels was $31 \%$. A similar prevalence was also found in three recent studies in patients with $\mathrm{PE}$ using different troponin assays: 18 (32\%) of 56 patients using a qualitative troponin T assay, ${ }^{4} 14$ (39\%) of 36 patients using a qualitative troponin I assay, ${ }^{3}$ and 43 (41\%) of 106 patients using a quantitative troponin I assay. ${ }^{2}$ In the cited and in the present study, cardiac troponin was a predictor of right ventricular dysfunction and adverse clinical outcomes in patients with PE.

In the present study, four of the 28 patients with a troponin I leak had preserved right ventricular function on echocardiography. Thus, right ventricular dysfunction should be confirmed in all troponin-positive PE patients with risk factors for coronary artery disease. Echocardiography is an important diagnostic and prognostic tool in patients with PE, and an increasing number of hospitals use echocardiography for risk stratification of their PE patients.

The present study is limited by the selection of adverse clinical outcomes used to define the combined end-point, including the need for thrombolysis and embolectomy. Ten of 13 patients who received thrombolysis fulfilled the criteria for clinically massive PE with shock or systemic hypotension according to the ESC guidelines. ${ }^{11}$ The remaining three patients with thrombolysis had submassive PE, defined as a preserved systolic arterial pressure and evidence of right ventricular dysfunction. According to the ESC guidelines, ${ }^{11}$ thrombolysis may also be considered in patients with submassive PE when no contraindications to thrombolysis are present. In contrast to MAPPET- $3,{ }^{12}$ the decision to initiate reperfusion therapy was based on the initial clinical presentation of the patient, particularly on shock index and echocardiographic findings, and not on secondary clinical deterioration. Thus, this study allows no conclusion about the prognostic value of echocardiography and troponin I in the prediction of secondary clinical deterioration in patients with elevated troponin I levels and right ventricular dysfunction who receive standard treatment with heparin alone.

\section{Conclusions}

In patients with acute PE, troponin I and echocardiography have an incremental prognostic value for adverse clinical outcomes including death, the need for cardiopulmonary resuscitation, mechanical ventilation, pressors, thrombolysis, catheter fragmentation, and surgical embolectomy. While troponin I measurements added most of the prognostic information for identifying highrisk patients, a normal echocardiogram combined with a negative troponin I level was most useful to identify patients at lowest risk for early death.

\section{References}

1. Goldhaber SZ, Visani L, De Rosa M. Acute pulmonary embolism: clinical outcomes in the International Cooperative Pulmonary Embolism Registry (ICOPER). Lancet 1999;353:1386-9.

2. Konstantinides S, Geibel A, Olschewski $M$ et al. Importance of cardiac troponins I and $\mathrm{T}$ in risk stratification of patients with acute pulmonary embolism. Circulation 2002;106:1263-8.

3. Meyer T, Binder L, Hruska $\mathrm{N}$ et al. Cardiac troponin I elevation in acute pulmonary embolism is associated with right ventricular dysfunction. J Am Coll Cardiol 2000;36:1632-6.

4. Giannitsis E, Muller-Bardorff M, Kurowski $V$ et al. Independent prognostic value of cardiac troponin $\mathrm{T}$ in patients with confirmed pulmonary embolism. Circulation 2000;102:211-7.

5. Remy-Jardin M, Remy J, Deschildre F et al. Diagnosis of pulmonary embolism with spiral CT: comparison with pulmonary angiography and scintigraphy. Radiology 1996;200:699-706. 
6. The PIOPED Investigators. Value of the ventilation/perfusion scan in acute pulmonary embolism. Results of the prospective investigation of pulmonary embolism diagnosis. JAMA 1990;263:2753-9.

7. Stein PD, Athanasoulis C, Alavi A et al. Complications and validity of pulmonary angiography in acute pulmonary embolism. Circulation 1992;85:462-8.

8. Goldhaber SZ. Echocardiography in the management of pulmonary embolism. Ann Intern Med 2002;136:691-700.

9. Ribeiro A, Lindmarker P, Juhlin-Dannfelt A et al. Echocardiography Doppler in pulmonary embolism: right ventricular dysfunction as a predictor of mortality rate. Am Heart J 1997;134:479-87.
10. Kucher N, Printzen G, Doernhoefer $\mathrm{T}$ et al. Novel management strategy for patients with suspected pulmonary embolism. Eur Heart J 2003;24:366-76.

11. Task Force on Pulmonary Embolism. Guidelines on diagnosis and management of acute pulmonary embolism. European Society of Cardiology. Eur Heart J 2000;21:1301-36.

12. Konstantinides S, Geibel A, Heusel G et al. Heparin plus alteplase compared with heparin alone in patients with submassive pulmonary embolism. N Engl J Med 2002;347:1143-50. 